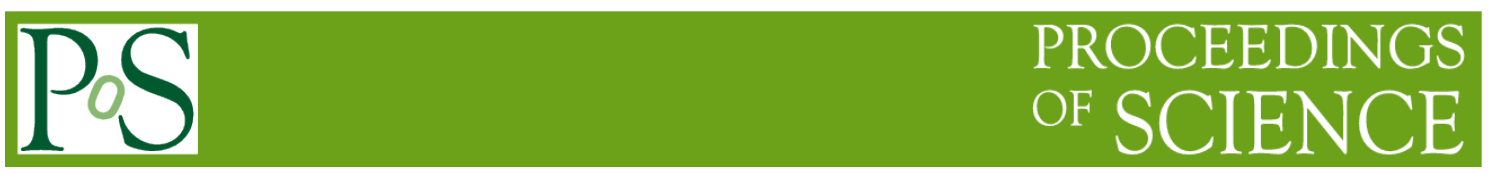

\title{
Simulation of Emergency Evacuation in School Building
}

\author{
Wenhui $\mathbf{L i}^{1}$ \\ Department of Computer Science and Technology,Jilin University, Changchun, 130012, China \\ Jinlong Zhu \\ Department of Computer Science and Technology, Jilin University,Changchun, 130012, China \\ E-mail: zhujinlong198409130126.com
}

\section{Huiying Li, Mingyu Sun,Yupeng Yang, Yunfan Du}

Department of Computer Science and Technology, Jilin University, Changchun,130012, China

The lack of crowd evacuation losses casualties and huge economic losses. The agent-based methods have been successfully employed by some scholars to reduce the loss by simulating behavior of pedestrian in case of emergency evacuation; but these models haven't not considered the people's psychological activity so that the simulating evacuation can not generate a practical evacuation plan. We proposed a model to simulate evacuation with Rule Base (KB) in a complex building architecture. The model employs a system of self-moving pedestrian intelligence models (SMPIM) whose motion is governed by the social-force model. KB contains the behavioral decision rules and the mental activity rules. The experiment shows the number of exits affects the total evacuation time. Based on the result analysis, the analysis of congestion bottleneck problem helps improve evacuation efficiency.

CENet2015

12-13 September 2015

Shanghai, China

\footnotetext{
${ }^{1}$ Speaker
} 


\section{Introduction}

The crowd evacuation is developed to calculate the escape time in the fire [1]. Researchers have studied many models used to simulate the crowd evacuation $[2,3]$. In all these models, the agent-based simulation is an important evacuation tool from the microscopic view because it covers important sub-problems ranging from the motion planning and the collision avoidance to the behavioral modeling. Agent regards evacuees as discrete entities with a level of artificial intelligence [4-6]. The agent-based modeling (ABM) approach simulates the real stress by the social-force model [7-9]. We adopt the Newton's equations to compute the moving of particle [10]; however, the agents are counted as independent entities acting from their own properties and the approach can't work out the optimal strategy for massive crowd evacuation in a complex scene.

These methods haven't considered the person's psychology for evacuation. We introduce the rule base as decision-making basis of people psychological activity and behavior in order to achieve more realistic simulation evacuation.

The paper structure is shown as follows. Section 2 is the agent model and defines two types of psychologies and three types of social force to affect the motion of pedestrians, which is followed by the Rule Base (RB) in Section 3. Section 4 presents the application of the methodology to the Computer Building in Jilin University. The final section contains the conclusion and the future work.

\section{Agent Model}

The agent-based modeling (ABM) has been used to simulate evacuation in a complex scene. Agents, as individuals, are endowed with human attributes, such as gender, age and walking speed. In this simulation, Fig. 1 shows a pedestrian as spherical agents. Each agent has a diameter $R_{2}$, which is a pre-collision detection area ranging from 0.5 to $0.7 \mathrm{~m} . \mathrm{R}_{3}$ is a comfortable distance ranging from 0.7 to $1 \mathrm{~m}$. The diameter is chosen randomly and it follows a uniform distribution. The variable $d_{i j}$ is the distance between two agents. When $d_{i j}$ is less than $\mathrm{R}_{2}$, they are regarded as a possible collision and their walking speed will be reduced. Everyone in simulation is randomly generated, its vertical projection area as shown in Table1.

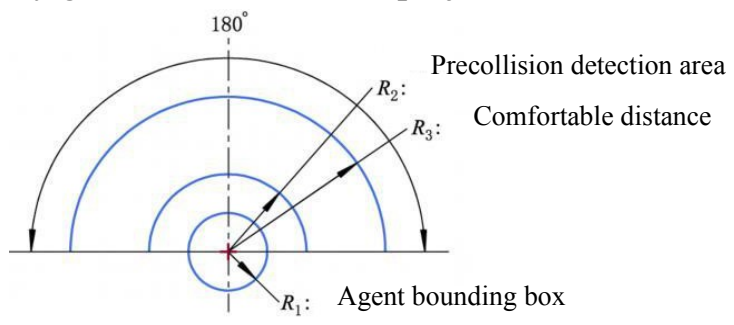

Figue1: Agent Information Perception Model

We propose a method to control the behavior each agent individually by a series of rules Table 2. Such simulation techniques are suitable for individual agents with inhomogeneous governing behaviors. As long as the agent detects the congestion degree of exit close to the current location being less than the threshold value, he/she will choose the export in order to escape. 


\begin{tabular}{llllllll}
\hline Gender & Body & $\mathrm{T} 1(\mathrm{~mm})$ & $\mathrm{T} 2(\mathrm{~mm})$ & $\mathrm{T} 3(\mathrm{~mm})$ & $\mathrm{T} 4(\mathrm{~mm})$ & $\mathrm{T} 5(\mathrm{~mm})$ & $\mathrm{T} 6(\mathrm{~mm})$ \\
\hline Male & Chest depth & 176 & 186 & 191 & 212 & 237 & 245 \\
& Across shoulder & 383 & 398 & 405 & 431 & 460 & 469 \\
\multirow{3}{*}{ Female } & Chest depth & 159 & 170 & 176 & 199 & 230 & 239 \\
& Across shoulder & 347 & 363 & 371 & 397 & 428 & 438 \\
\cline { 2 - 7 }
\end{tabular}

Table 1: Human Dimensions of Chinese Adults

When people meet any danger, the evacuees can have fear, which can affect the behaviors of evacuation, such as lower speed. The psychology prompts the agent to slow down so that the agent will not be able to escape. For the appraisal of panic psychology, we present a panic coefficient according to the personnel's psychological quality value and risk value. If the panic coefficient is greater than the threshold, the agent is in a panic state. The rational value of agent is reduced so that arithmetic reduces knowledge base decision depth.

The Psychology rescue refers to evacuation to help a weakling for evacuation. When a person's walking speed is too low due to his/her own attribute or panic, his/her relatives or friends will help them improve the speed. If the acquaintance degree of two agents is greater than the nominal threshold, the two agents are an aid pair. Relationship of aid pair is 1-N relationship. Where 1 is the aid recipients, $\mathrm{N}$ is the rescuer, $\mathrm{N}<3$.

Three kinds of social forces contain the motivational force, the powerful repulsive tendency and the inferior repulsive tendency.

Motivational force describes the psychological tendency of a pedestrian to escape to the destination. It is a measure of motivation and the decision of the pedestrian to perform certain movements. The repulsive tendency is a force between two pedestrians, and it includes two types, power repulsive tendency and inferior repulsive tendency. The direction of this force is the opposite direction of moving in the center line of two pedestrians.

\section{Rule Base}

In the computer science, the rule base is a data source to record rules of nature. According to some rule of behaviors of pedestrians, we format the rules to semantic information, that is to say, the semantic information is operating constraints and operating. The rule-based programming attempts to derive execution instructions from a starting set of data and rules. We use Rule Base as an agent's behavior decision-making basis, where the agent's behavior including psychological activity and motion. We define ten simple rule patterns for simulating the pedestrian's behaviors.

\begin{tabular}{|c|c|}
\hline ID & Description of a rule \\
\hline $\mathrm{R}_{1}$ & $\begin{array}{l}\text { If dij }<\mathrm{R}_{2 \mathrm{i}}+\mathrm{R}_{2 \mathrm{j}} \text {, set powerful repulsive tendency between Agent } \mathrm{i}_{\mathrm{i}} \text { and } \\
\text { Agent } \mathrm{j}_{\mathrm{j}}\end{array}$ \\
\hline $\mathrm{R}_{2}$ & $\begin{array}{l}\text { If dij }<\mathrm{R}_{3 \mathrm{i}}+\mathrm{R}_{3 \mathrm{j}} \text {, set inferior repulsive tendency between } \text { Agent }_{\mathrm{i}} \text { and } \\
\text { Agent } \\
\text {. }\end{array}$ \\
\hline $\mathrm{R}_{3}$ & $\begin{array}{l}\text { If s1-s2>e (nominal threshold of speed different), s1 is speed of Agent A } \\
\text { and } \mathrm{s} 2 \text { is speed of Agent B, A is behind B. Then A will outstrip B. }\end{array}$ \\
\hline $\mathrm{R}_{4}$ & $\begin{array}{l}\text { A subject chooses the exit with the fewer agents for evacuation in } \\
\text { information perception layer. }\end{array}$ \\
\hline $\mathrm{R}_{5}$ & If obstacles block in the escape routes, a subject jumps over obstacles. \\
\hline
\end{tabular}

Table 2: Rule Base 
Fig. 2 shows the self-moving pedestrian intelligence models that the individual agent will extract perceive information; then the system analyzes the perception information as the input parameters of Inference engine; finally, the inference engine outputs the motion sequence according to the behavioral model.

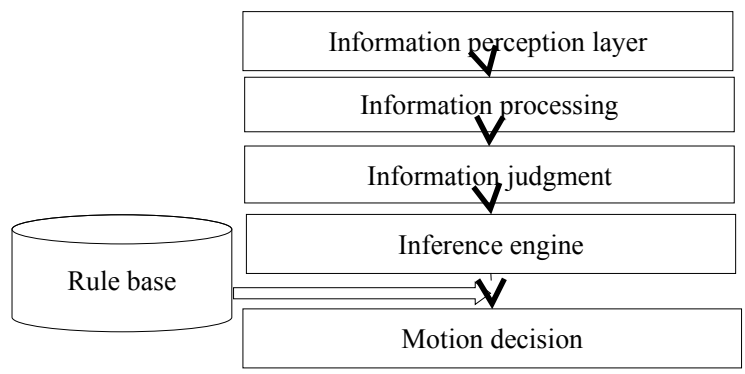

Figure 2: Self-moving Pedestrian Intelligence Models

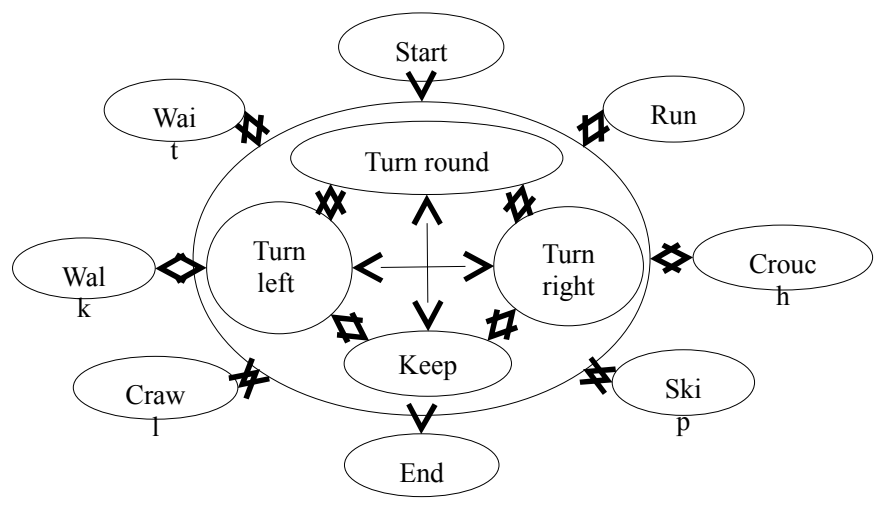

Figure 3: Motion Model

We proposed a behavior model consisting of ten simple behaviors in Fig. 3. We put the behavior model as a diagram, the node is the behavior and the edge is the association of behavior. The behavior transforms another behavior by edge connection association in Fig.3. For example, if the obstacles block the escape routes, an agent jumps over such obstacles. The motion sequence of the process is walking, jumping obstacles and walking. Its corresponding motion model state is Walk, Keep, Skip, Keep and Walk. We can see the transition state through the four basic actions of the center between the eight moves around. 


\section{Experiment Study}

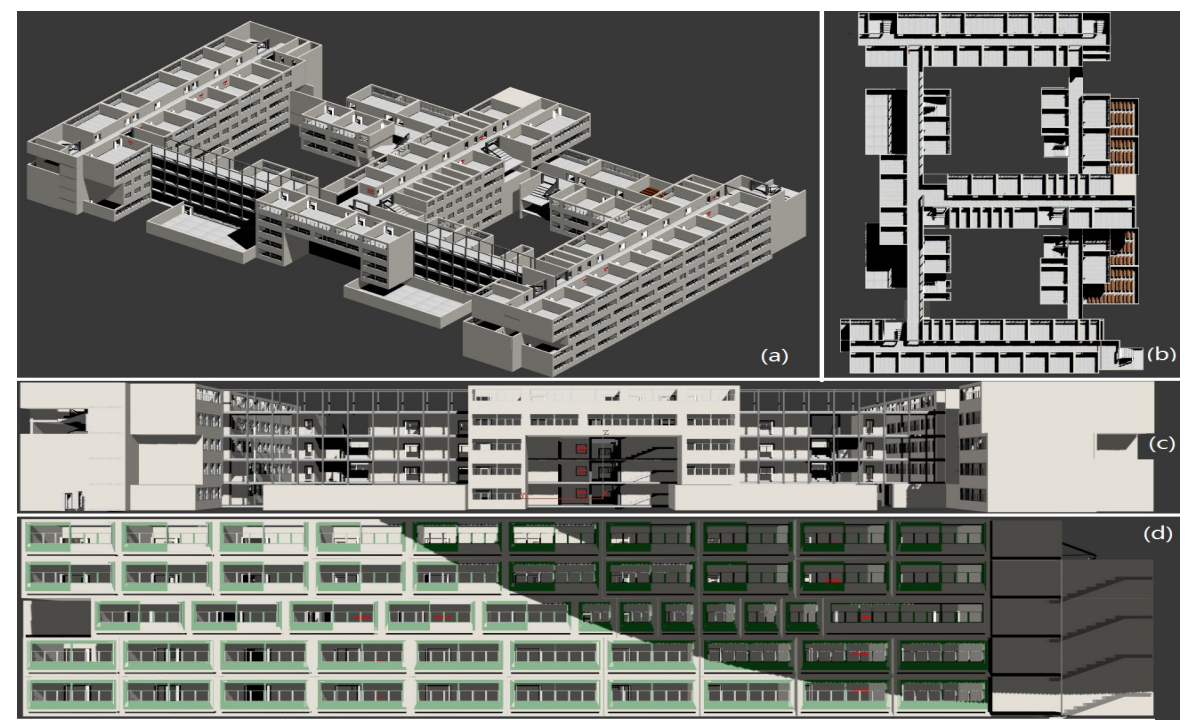

Figure 4: Layout Plan

\begin{tabular}{llll}
\hline Items & Headcount & Items & Headcount \\
\hline 1 floor total number of people & 806 & Room S3 & 6 \\
2 floor total number of people & 804 & Room S4 & 7 \\
3 floor total number of people & 444 & Room S5 & 8 \\
4 floor total number of people & 444 & Room M1 & 10 \\
5 floor total number of people & 114 & Room M2 & 35 \\
Room S1 & 2 & Room B1 & 60 \\
Room S2 & 5 & Room B2 & 100 \\
\hline
\end{tabular}

Table 3: People Number of Buildings for Distribution

\begin{tabular}{ll}
\hline Items & Size $(\mathrm{m})$ \\
\hline Floor height & 3.3 \\
Width of alleyway(max) & 5.5 \\
Width of alleyway(min) & 3.7 \\
Width of single leaf door & 1.2 \\
Width of double swing door & 2.2 \\
\hline
\end{tabular}

Table 4: Building Specifications

In the experiment, we use the computer building in Jilin University as the object of an experiment to evaluate the path value at risk. Shown in Fig. 4, (a) is panorama, (b) is top view, (c) is elevation drawing and (d) is side view. The building specifications and people distribution are shown in Table 3 and Table 4 respectively.

Fig. 4 shows the distribution of the exit of the first floor. Exit 2 and Exit 4 are of double swing door, Exit 1 and Exit 3 is of single leaf door. The width of alleyway connection Exit 1 and Exit 3 is of $3.7 \mathrm{~m}$, the width of other alleyway is of $5.5 \mathrm{~m}$. The first floor and second floor respectively have two amphitheaters whose agents' capacity number is 100 . The outflow coefficient is the pedestrian number of evacuation by unit exit width and unit time. The outflow of Exit 1 and Exit 3 are 2, the outflow of Exit 2 and Exit 4 are 4.

We analyze the simulation results of evacuation only to find Exit 4 is the most serious congestion area. The majority corridors share Exit 4, which is leading to a large number of 
pedestrians gathered here for evacuation. Fig. 6 shows the pedestrians number distribution of each exit.

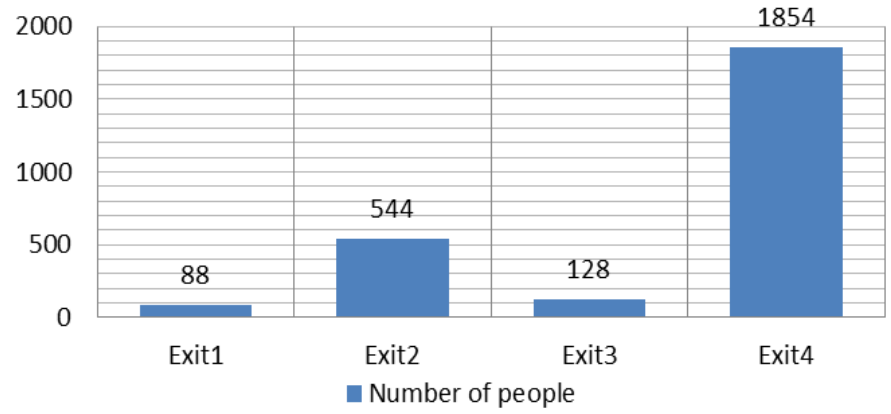

Figure 5: Pedestrians' Distribution

We analyze the evacuation data of Exit 4 because it is the most serious area traffic congestion. There are two types of data analysis patterns. See Fig. 7 for the congestion change curve and Fig. 8 for the evacuation analysis of pedestrians on each floor.

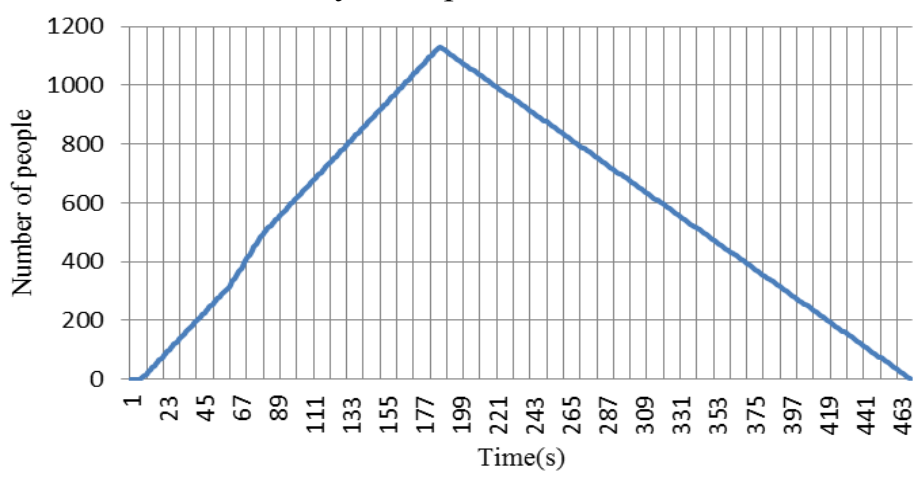

Figure 6: Congestion Distribution

The evacuation distribution shows that the first floor and second floor contain so many pedestrians, which prolong the evacuation time. We suggest that pedestrians on the $3^{\text {rd }}$ floor, $4^{\text {th }}$ floor and $5^{\text {th }}$ floor for escaping from other exit so that the congestion degree and the evacuation time can be reduced to the minimum.

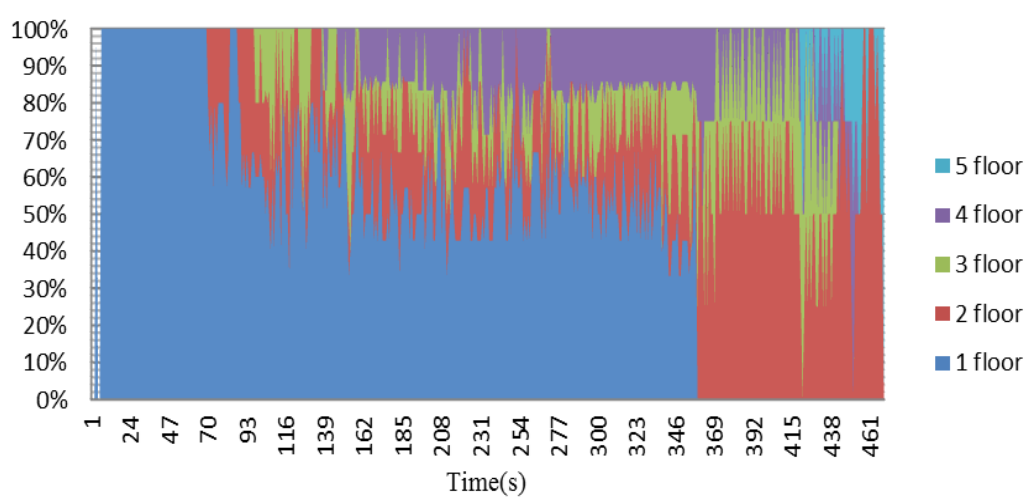

Figure 7: Evacuation Distribution

\section{Conclusion}

This article has developed an agent-model-based method to simulate the large crowd evacuation. We combine the agent model and the rule base model to simulate the pedestrian's 
behavior sin the fire. The pedestrian behavior chooses ten typical motions (wait, keep, turn left, turn right, run, walk, skip, crouch, scrawl and turn round) as the motion model. We use the rule base method to deduce behavior and reaction according to the environment in order to simulate the real environment. We propose the method to prove our simulation credibility through the test data. Further studies should be carried out to better the rule base by machine learning and be combined with video detection technology for the real environment interaction.

\section{References}

[1] Jason Sewall, David Wilkie and Ming Lin. Interactive Hybrid Simulation of Large-Scale Traffic[J]. ACM TRANSACTIONS ON GRAPHICS. 30(6): 1-18(2012).

[2] Xiaoshan Pan, Charles S. Han, K. Dauber, Ken and Kincho H. Law. Human and social behavior in computational modeling and analysis of egress [J]. Automation in Construction. 15(4): 448461(2006).

[3] Ayse I. Parlak, James H. Lambert, Thomas M. Guterbock and Janet L. Clements. Population behavioral scenarios influencing radiological disaster preparedness and planning[J]. Accident Analysis and Prevention. 48(2): 353-362(2012).

[4] S Hoogendoorn and PHL Bovy. Simulation of pedestrian flows by optimal control and differential games[J]. Optimal Control Applications \& Methods. 24(3): 153-172(2003).

[5] Bonabeau. Agent-based modeling: methods and techniques for simulating human systems[J]. Proceedings of the National Academy of Sciences of the United States of America. 99(3) : 72807287 (2002).

[6] Xiaoping Zheng, Wei Li and Chao Guan. Simulation of evacuation processes in a square with a partition wall using a cellular automaton model for pedestrian dynamics[J]. Physica a-Statistical Mechanics and its Applications. 389(11) : 2177-2188 (2010).

[7] Ha Vi, Lykotrafitis and George. Agent-based modeling of a multi-room multi-floor building emergency evacuation[J]. Physica A-statistical Mechanics And Its Applications. 391(8) : 27402751( 2012).

[8] Chooramun Nitish, Lawrence Peter and Galea Edwin. An agent based evacuation model utilising hybrid space discretization discretisation[J]. Safey Science. 50(8) : 1685-1694( 2012).

[9] Manley Matthew and Kim Yong Seog. Modeling emergency evacuation of individuals with disabilities (exitus): An agent-based public decision support system [J]. Expert Systems With Applications. 39(9) : 8300-8311(2012).

[10] Dirk Helbing, Peter Molnar, Illés J Farkas and Kai Bolay. Self-organizing pedestrian movement $[\mathrm{J}]$. Environ. Plan. B,Planning and Design. 28(10) :361-383( 2001). 